\title{
Analysis of Antioxidant Activity of Chinese Brown Rice by Fourier-Transformed Near Infrared Spectroscopy and Chemometrics
}

\author{
Xianshu Fu, Xiaoping Yu, Zihong Ye, and Haifeng Cui \\ Zhejiang Provincial Key Laboratory of Biometrology and Inspection \& Quarantine, College of Life Sciences, \\ China Jiliang University, Hangzhou 310018, China
}

Correspondence should be addressed to Xiaoping Yu; lhl740901@163.com

Received 14 August 2014; Accepted 11 October 2014

Academic Editor: Hai-Yan Fu

Copyright (C) 2015 Xianshu Fu et al. This is an open access article distributed under the Creative Commons Attribution License, which permits unrestricted use, distribution, and reproduction in any medium, provided the original work is properly cited.

\begin{abstract}
This paper develops a rapid method using near infrared (NIR) spectroscopy for analyzing the antioxidant activity of brown rice as total phenol content (TPC) and radical scavenging activity by DPPH (2,2-diphenyl-2-picrylhydrazyl) expressed as gallic acid equivalent (GAE). Brown rice $(n=121)$ collected from five producing areas was analyzed for TPC and DPPH by reference methods. The NIR reflectance spectra were measured with compact powders of samples and no treatment was used. Full-spectrum partial least squares (FS-PLS) and interval PLS (iPLS) were used as the regression methods to relate the antioxidant activity values to the NIR data. The spectral range of $4800-5600 \mathrm{~cm}^{-1}$ plus $6000-6400 \mathrm{~cm}^{-1}$ has the best correlation with TPC, while the range of $4400-5200 \mathrm{~cm}^{-1}$ plus $6000-6400 \mathrm{~cm}^{-1}$ is the most suitable for predicting DPPH. With standard normal variate (SNV) transformation and the selected wavelength ranges, the root mean squared error of prediction (RMSEP) is $0.062 \mathrm{mg} \mathrm{GAE} \mathrm{g}^{-1}$ for TPC and $0.141 \mathrm{mg} \mathrm{GAE} \mathrm{g}^{-1}$ for DPPH radical, respectively. The multiple correlation coefficients of predictions for TPC and DPPH are 0.962 and 0.974 , respectively. The developed NIR method might have a potential application to quality control of brown rice in the domestic market.
\end{abstract}

\section{Introduction}

As one of the oldest domesticated grains, rice serves as the staple food for half of the global population. China is the first leading producer of rice in the entire world [1] and there are lots of varieties of rice cultivated in China. Brown rice has attracted much attention in recent years for its special taste and healthy effects $[2,3]$. Compared with nonbrown rice, brown rice varieties not only provide high-quality protein, high fiber and vitamin contents, but also have a much more higher content of antioxidative compounds, including anthocyanidins (aglycones), cyanidin and malvidin [4]; polymeric procyanidins [5]; the phenolic compounds: anisole, protocatechuic acid methyl ester, syringaldehyde, and vanillin [6-9]; the phenolic compounds ferulic and sinapinic acids. A more detailed review of the antioxidative compounds is referred to in [3]. Brown rice has the potential to improve human health because the antioxidative compounds have the ability to inhibit the formation or to reduce the concentrations of reactive cell-damaging free radicals.

The contents of antioxidative compounds in brown rice mainly depend on the varieties, cultivation conditions, and processing. It is well known that long-term storage can degrade the quality of brown rice because of oxidation. In China, for economic reasons, it is profitable to sell degraded or fraud brown rice by extracting pigments and other active components before putting them on the supermarket shelf. Therefore, it is necessary to develop a rapid and reliable method for determining the quality of brown rice, especially its antioxidant activity.

Traditional methods for evaluating antioxidant activity generally can fall into two classes: direct determination of antioxidant capacity and determination of the levels of the main antioxidant components [10]. The methods for direct determination include ferric reducing/antioxidant power (FRAP), 2,2' -azino-bis(3-ethylbenzthiazoline-6-sulfonic acid) 
(ABTS), Trolox equivalent antioxidant capacity (TEAC), 2,2-diphenyl-1-picrylhydrazyl (DPPH), and oxygen radical absorbance capacity (ORAC) [11]. For determining the contents of antioxidant components, high performance liquid chromatography (HPLC), high performance capillary electrophoresis (HPCE), and colorimetric determination have been used and the antioxidant components analyzed include flavone C-glucosides, total flavonoids, and total phenolic content (TPC) $[12,13]$. However, the above methods are time consuming, laborious, and inconvenient to use considering the large number of samples to be analyzed.

Compared with traditional methods, NIR spectroscopy has many advantages including less sample preparation, reduced analysis time and cost. Therefore, NIR has been widely used for rapid analysis of antioxidant activity in various food products [14]. In this work, we investigated the feasibility of using NIR spectroscopy for rapid analysis of the antioxidant activity of brown rice in Chinese market. The objectives of this paper include (1) developing a quantitative model between the NIR spectra and two antioxidant activity indexes, namely, TPC and DPPH; (2) selecting useful wavelength intervals by interval partial least squares [15]; (3) comparing the predictive performances of calibration models based on different data preprocessing methods.

\section{Materials and Methods}

2.1. Brown Rice. Brown rice samples were collected from domestic markets and the producing areas are Yunnan (25), Hunan (21), Sichuan (27), Guizhou (25), and Shanxi (23). All the 121 samples are harvested and analyzed in 2013. All the samples were cleaned and stored at $25^{\circ} \mathrm{C}$ before analysis.

\subsection{Analysis of Antioxidant Activities}

2.2.1. Sample Preparation. The method in [16] was used. About $10 \mathrm{~g}$ of rice sample was ground by a crusher and extracted with $100 \mathrm{~mL}$ of mixture solvent (acetone: water, $75: 25 \mathrm{v} / \mathrm{v}$ ) for $1.5 \mathrm{~h}$ at $25^{\circ} \mathrm{C}$. The extracts were then centrifuged at $950 \mathrm{~g}$ for $15 \mathrm{~min}$. The supernatant was then kept at $-20^{\circ} \mathrm{C}$ for further analysis.

\subsubsection{Reference Analysis of Total Phenolic Content (TPC). A} modified version of the Folin-Ciocalteu assay [17] was used to determine the TPC values for the prepared extracts. For the analysis, $20 \mu \mathrm{L}$ of extract, gallic acid standard, and blank were analyzed in parallel. Firstly, $1.58 \mathrm{~mL}$ of distilled water was added, followed by $100 \mu \mathrm{L}$ of Folin-Ciocalteu reagent. The mixtures were fully mixed and within $8 \mathrm{~min}, 300 \mu \mathrm{L}$ of sodium carbonate was added. The mixtures were magnetically stirred and allowed to incubate in dark for $30 \mathrm{~min}$ at $40^{\circ} \mathrm{C}$. The absorbance at $765 \mathrm{~nm}$ was measured. The TPC values of samples were computed using the standard curve of aqueous gallic acid solutions and the unit was $\mathrm{mg} \mathrm{GAE} / \mathrm{g}$.

2.2.3. Reference Analysis of DPPH. The DPPH (2,2-diphenyl1-picrylhydrazyl) radical scavenging activity of the extracts was measured using the method in [18]. Firstly, the blank was prepared by adding $100 \mu \mathrm{L}$ methanol to $1.4 \mathrm{~mL}$ of $\mathrm{DPPH}$ radical methanolic solution $\left(10^{-4} \mathrm{M}\right)$. Separately, $100 \mu \mathrm{L}$ of the prepared extract was added to $1.4 \mathrm{~mL}$ of DPPH radical methanolic solution. The absorbance at $517 \mathrm{~nm}$ was measured.

2.2.4. NIR Analysis. The NIR diffuse reflectance spectra of all the samples were collected in $4000-12000 \mathrm{~cm}^{-1}$ on a BrukerTENSOR37 FTIR system (Bruker Optics, Ettlingen, Germany) using OPUS software. All the spectra were measured with a $\mathrm{PbS}$ detector and an internal gold background as the reference. The resolution was $4 \mathrm{~cm}^{-1}$ and the scanning interval was $1.929 \mathrm{~cm}^{-1}$. Therefore, each spectrum had 4148 wavelengths. The scanning number was 64 , because more scans did not reduce the signal-to-noise ratio significantly.

2.3. Chemometrics. To obtain a set of representative objects for training/validating the calibration models, the DUPLEX algorithm [19] was used to split the measured objects into two data sets, one for training and the other for validation. DUPLEX alternatively picks up the two furthest objects in the objects pool for the training set and test set.

The quantitative modeling was performed using PLS and iPLS [15]. The iPLS algorithm was used to select informative spectral intervals for predicting TPC and DHHP. The principle of iPLS is to split the full spectra into smaller equidistant regions and, afterwards, build separate PLS regression models for each subinterval, using the same number of latent variables. Thereafter, a modeling error is calculated for each subinterval and for the full-spectrum model. The regions with the lowest error are selected and combined to build a final PLS model. An advantage of iPLS is that it can represent the predictive ability of each interval in a graphical display and enable a fast and reasonable selection of spectral intervals.

\section{Results and Discussions}

The content ranges reference values of antioxidant activity in calibration samples were 1.0933-6.0464 for TPC and 0.78335.8125 (mg GAE/g) for $\mathrm{DPPH}$, respectively. The content ranges of TPC and DPPH in validation samples were 1.21355.9406 and $0.8121-5.4903$ (mg GAE/g), respectively. This indicates that the DUPLEX method can split the data properly for calibration and validation. To reduce the unwanted spectral variations, three preprocessing methods, including smoothing, taking second-order derivative (D2) [20], and standard normal variate (SNV) transformation [21], were applied to the raw data. The raw NIR spectra and smoothed, D2, SNV spectra are shown in Figure 1. As seen from Figure 1, both D2 and SNV can reduce shifts caused by backgrounds. The DUPLEX algorithm was performed on the raw data to divide the 121 objects into a training set of 80 samples and a test set of 41 samples. For both full-spectrum PLS (FS-PLS) and iPLS models, leave-one-out cross-validation (LOOCV) was used to estimate model complexity and the errors of both FS-PLS and iPLS models were estimated by root mean squared error of cross-validation (RMSECV). The calibration and prediction results of FP-PLS models are listed in Table 1.

For iPLS models, the total spectral range was sequentially (from $4000 \mathrm{~cm}^{-1}$ to $12000 \mathrm{~cm}^{-1}$ ) split into 20 spectral 
TABLE 1: Results of full-spectrum PLS (FS-PLS) models for predictions of antioxidant activity of brown rice.

\begin{tabular}{|c|c|c|c|c|c|c|}
\hline & \multicolumn{3}{|c|}{ TPC (mg GAE/g) } & \multicolumn{3}{|c|}{ DPPH (mg GAE/g) } \\
\hline & RMSECV $^{\mathrm{a}}$ & RMSEP $^{\mathrm{b}}$ & $\mathrm{LVs}^{\mathrm{c}}$ & RMSECV & RMSEP & LVs \\
\hline Raw data & 0.242 & 0.303 & 7 & 0.269 & 0.283 & 8 \\
\hline Smoothing & 0.196 & 0.232 & 6 & 0.243 & 0.244 & 9 \\
\hline D2 & 0.191 & 0.209 & 6 & 0.233 & 0.219 & 8 \\
\hline SNV & 0.162 & 0.167 & 6 & 0.199 & 0.211 & 7 \\
\hline
\end{tabular}

${ }^{a}$ RMSECV: root mean squared error of cross-validation.

${ }^{b}$ RMSEP: root mean squared error of prediction.

${ }^{\mathrm{c}}$ Number of PLS components.
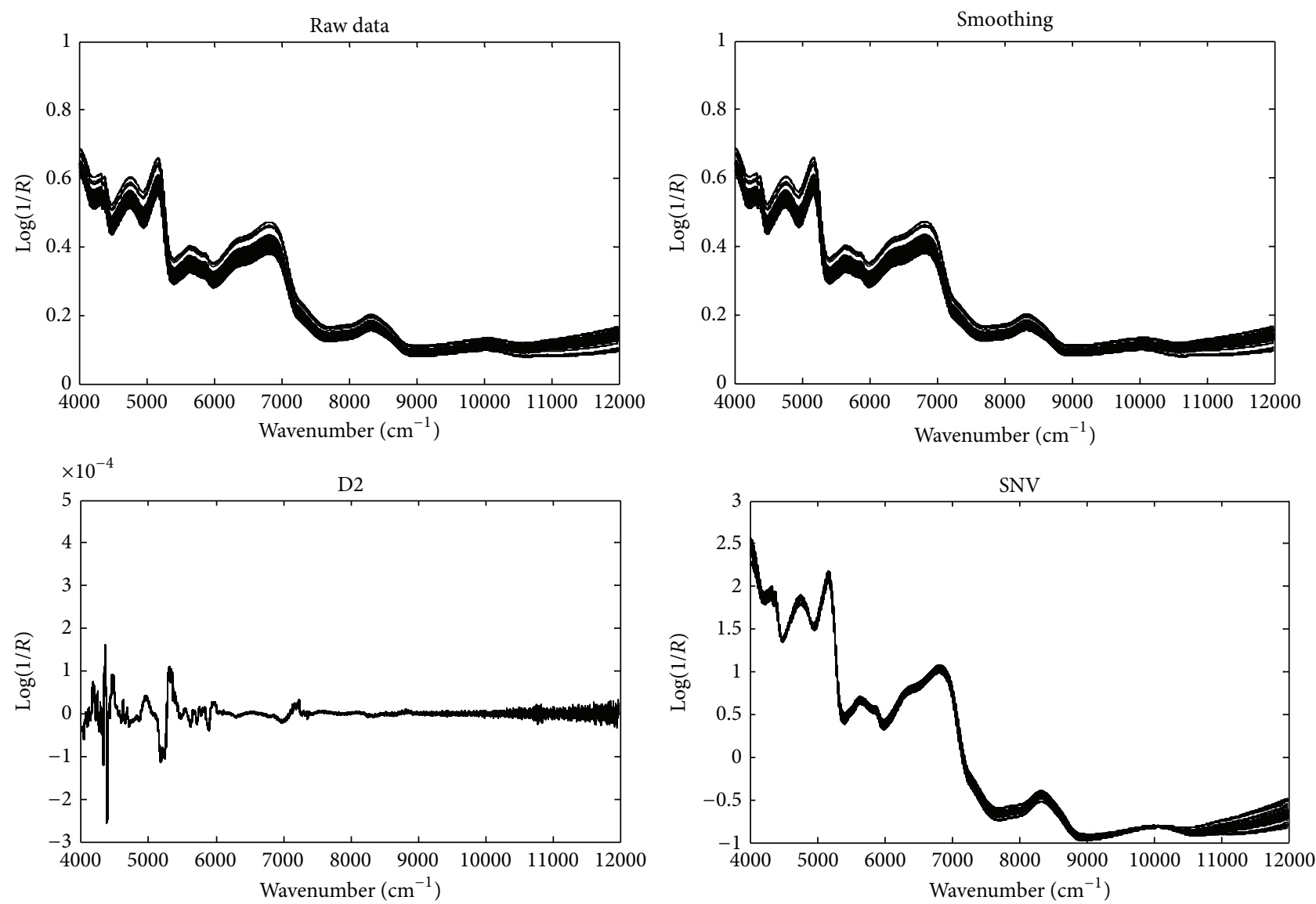

FIGURE 1: The raw NIR spectra and the smoothed, second-order derivative (D2), and standard normal variate (SNV) spectra of brown rice objects.

intervals with an equal width of $400 \mathrm{~cm}^{-1}$. At each interval, a PLS model is built to predict TPC and DPPH values. The numbers of PLS components were determined to obtain the lowest RMSECV. With each data preprocessing method, three intervals with the lowest RMSECV values were selected and combined to build the final iPLS model. The spectral intervals selected for predictions of TPC and DPPH are listed in Table 2. The most accurate interval models were obtained by SNV preprocessing. With standard normal variate (SNV) transformation, the root mean squared error of prediction (RMSEP) is $0.062 \mathrm{mg} \mathrm{GAE} \mathrm{g}^{-1}$ for TPC (selected spectral intervals, $4800-5600 \mathrm{~cm}^{-1}$ and $\left.6000-6400 \mathrm{~cm}^{-1}\right)$ and $0.141 \mathrm{mg} \mathrm{GAE} \mathrm{g}^{-1}$ for DPPH radical (selected spectral intervals, $4400-5200 \mathrm{~cm}^{-1}$ and $\left.6000-6400 \mathrm{~cm}^{-1}\right)$, respectively.
The correction coefficients $(r)$ of predictions for TPC and DPPH are 0.962 and 0.974 , respectively. The RMSECV for each spectral interval with SNV for predictions of TPC and DPPH is shown in Figure 2. The results indicate that iPLS can effectively select useful spectral intervals for predicting TPC and DPPH. By comparing the results in Tables 1 and 2, wavelength selection can improve the model accuracy more significantly than data preprocessing, indicating that uninformative wavelengths can degrade the FS-PLS model.

\section{Conclusions}

A rapid method for determination of antioxidant activity was developed by near infrared (NIR) spectroscopy and 
TABLE 2: Results of interval partial least squares (iPLS) models for predictions of antioxidant activity of brown rice.

\begin{tabular}{|c|c|c|c|c|c|c|}
\hline & \multicolumn{3}{|c|}{ TPC (mg GAE/g) } & \multicolumn{3}{|c|}{ DPPH (mg GAE/g) } \\
\hline & Selected intervals & RMSEP $^{\mathrm{a}}$ & $\mathrm{LVs}^{\mathrm{b}}$ & Selected intervals & RMSEP & LVs \\
\hline Raw data & $3,6,8$ & 0.179 & 4 & $2,5,6$ & 0.175 & 6 \\
\hline Smoothing & $3,4,8$ & 0.131 & 5 & $2,4,6$ & 0.188 & 4 \\
\hline $\mathrm{D} 2$ & $2,4,5$ & 0.117 & 4 & $1,3,4$ & 0.181 & 4 \\
\hline SNV & $3,4,6$ & 0.062 & 4 & $2,3,6$ & 0.141 & 5 \\
\hline
\end{tabular}

${ }^{a}$ RMSEP: root mean squared error of prediction by the final iPLS with selected spectral intervals.

${ }^{b}$ Number of components of the final iPLS models including all the selected spectral intervals.
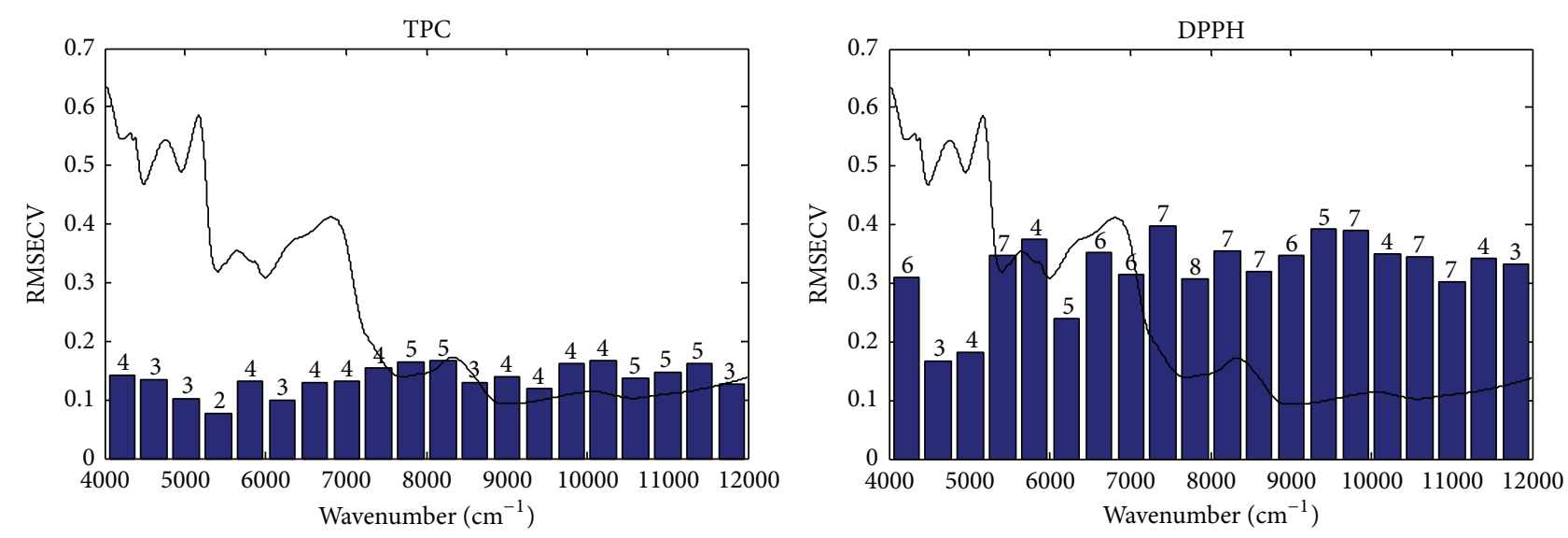

FIGURE 2: Root mean squared errors of cross-validation (RMSECV) obtained for each spectral interval by interval partial least squares (iPLS) with standard normal variate (SNV). The number at each bar indicates the number of PLS components at each wavelength interval.

chemometrics. By comparison of the results by FS-PLS and iPLS, wavelength selection can significantly improve the calibration accuracy of TPC and DPPH. The most suitable data preprocessing method was SNV. With SNV transformation and the selected wavelength ranges, the RMSEP is $0.062 \mathrm{mg} \mathrm{GAE} \mathrm{g}^{-1}$ for TPC and $0.141 \mathrm{mg} \mathrm{GAE} \mathrm{g}^{-1}$ for DPPH radical, respectively. The proposed method will provide a useful alternative tool to the physical and chemical analysis methods for brown rice.

\section{Conflict of Interests}

The authors declare that there is no conflict of interests regarding the publication of this paper.

\section{Acknowledgments}

The authors are grateful for the financial support from the Public Welfare Social Development Project of Zhejiang Province (no. 2013C33032), the National Public Welfare Industry Project of China (no. 201210092, 2012104019), and Zhejiang Province Department of Education Fund Item (no. Y201122027).

\section{References}

[1] Y. S. Savitha and V. Singh, "Status of dietary fiber contents in pigmented and non-pigmented rice varieties before and after parboiling," LWT_Food Science and Technology, vol. 44, no. 10, pp. 2180-2184, 2011.

[2] S. Saikia, H. Dutta, D. Saikia, and C. L. Mahanta, "Quality characterisation and estimation of phytochemicals content and antioxidant capacity of aromatic pigmented and non-pigmented rice varieties," Food Research International, vol. 46, no. 1, pp. 334-340, 2012.

[3] S. H. Nam, S. P. Choi, M. Y. Kang, H. J. Koh, N. Kozukue, and M. Friedman, "Antioxidative activities of bran extracts from twenty one pigmented rice cultivars," Food Chemistry, vol. 94, no. 4, pp. 613-620, 2006.

[4] J. W. Hyun and H. S. Chung, "Cyanidin and malvidin from Oryza sativa cv. heugjinjubyeo mediate cytotoxicity against human monocytic leukemia cells by arrest of $\mathrm{G}_{2} / \mathrm{M}$ phase and induction of apoptosis," Journal of Agricultural and Food Chemistry, vol. 52, no. 8, pp. 2213-2217, 2004.

[5] T. Oki, M. Masuda, M. Kobayashi et al., "Polymeric procyanidins as radical-scavenging components in red-hulled rice," Journal of Agricultural and Food Chemistry, vol. 50, no. 26, pp. 7524-7529, 2002.

[6] A. M. Asamarai, P. B. Addis, R. J. Epley, and T. P. Krick, "Wild rice hull antioxidants," Journal of Agricultural and Food Chemistry, vol. 44, no. 1, pp. 126-130, 1996.

[7] F. D. Goffman and C. J. Bergman, "Rice kernel phenolic content and its relationship with antiradical efficiency," Journal of the Science of Food and Agriculture, vol. 84, no. 10, pp. 1235-1240, 2004.

[8] S. C. Lee, J. H. Kim, S. M. Jeong et al., "Effect of far-infrared radiation on the antioxidant activity of rice hulls," Journal of 
Agricultural and Food Chemistry, vol. 51, no. 15, pp. 4400-4403, 2003.

[9] M. Miyazawa, T. Oshima, K. Koshio, Y. Itsuzaki, and J. Anzai, "Tyrosinase inhibitor from black rice bran," Journal of Agricultural and Food Chemistry, vol. 51, no. 24, pp. 6953-6956, 2003.

[10] D. Wu, J. Chen, B. Lu, L. Xiong, Y. He, and Y. Zhang, "Application of near infrared spectroscopy for the rapid determination of antioxidant activity of bamboo leaf extract," Food Chemistry, vol. 135, no. 4, pp. 2147-2156, 2012.

[11] F. Saura-Calixto, J. Pérez-Jiménez, S. Arranz et al., "Updated methodology to determine antioxidant capacity in plant foods, oils and beverages: extraction, measurement and expression of results," Food Research International, vol. 41, no. 3, pp. 274-285, 2008.

[12] Y. Zhang, B. Bao, B. Lu, Y. Ren, X. Tie, and Y. Zhang, "Determination of flavone $\mathrm{C}$-glucosides in antioxidant of bamboo leaves (AOB) fortified foods by reversed-phase high-performance liquid chromatography with ultraviolet diode array detection," Journal of Chromatography A, vol. 1065, no. 2, pp. 177-185, 2005.

[13] Y. Zhang, X.-Q. Wu, and Z.-Y. Yu, "Comparison study on total flavonoid content and anti-free redical activity of the leaves of bamboo, phyllostachys nigra, and Ginkgo bilabo," China Journal of Chinese Materia Medica, vol. 27, no. 4, pp. 254-320, 2002.

[14] X. Lu and B. A. Rasco, "Determination of antioxidant content and antioxidant activity in foods using infrared spectroscopy and chemometrics: a review," Critical Reviews in Food Science and Nutrition, vol. 52, no. 10, pp. 853-875, 2012.

[15] L. Nørgaard, A. Saudland, J. Wagner, J. P. Nielsen, L. Munck, and S. B. Engelsen, "Interval partial least-squares regression (iPLS): a comparative chemometric study with an example from nearinfrared spectroscopy," Applied Spectroscopy, vol. 54, no. 3, pp. 413-419, 2000.

[16] E. Atala, L. Vásquez, H. Speisky, E. Lissi, and C. López-Alarcón, "Ascorbic acid contribution to ORAC values in berry extracts: an evaluation by the ORAC-pyrogallol red methodology," Food Chemistry, vol. 113, no. 1, pp. 331-335, 2009.

[17] S. Slinkard and V. L. Singleton, "Total phenol analysis: automation and comparison with manual methods," American Journal of Enology and Viticulture, vol. 28, pp. 49-55, 1977.

[18] W. Brand-Williams, M. E. Cuvelier, and C. Berset, "Use of a free radical method to evaluate antioxidant activity," Food Science and Technology, vol. 28, no. 1, pp. 25-30, 1995.

[19] R. D. Snee, "Validation of regression models, methods and examples," Technometrics, vol. 19, pp. 415-428, 1977.

[20] A. Savitzky and M. J. E. Golay, "Smoothing and differentiation of data by simplified least squares procedures," Analytical Chemistry, vol. 36, no. 8, pp. 1627-1639, 1964.

[21] R. J. Barnes, M. S. Dhanoa, and S. J. Lister, "Standard normal variate transformation and de-trending of near-infrared diffuse reflectance spectra," Applied Spectroscopy, vol. 43, no. 5, pp. 772777, 1989. 

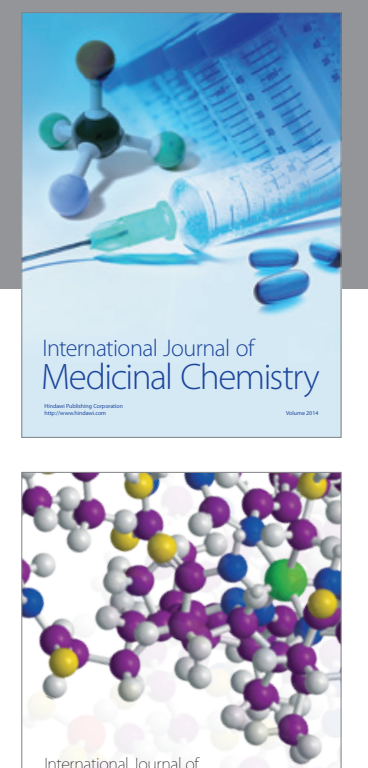

\section{Carbohydrate} Chemistry

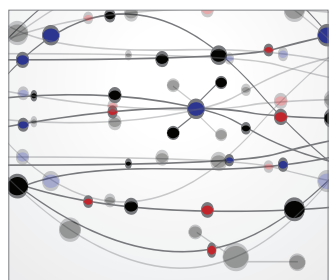

The Scientific World Journal
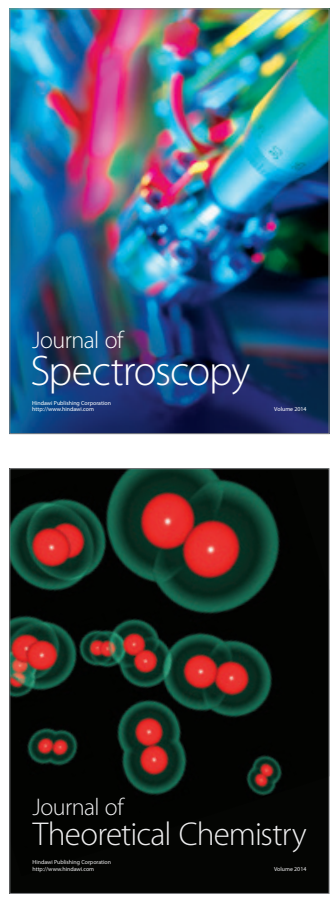
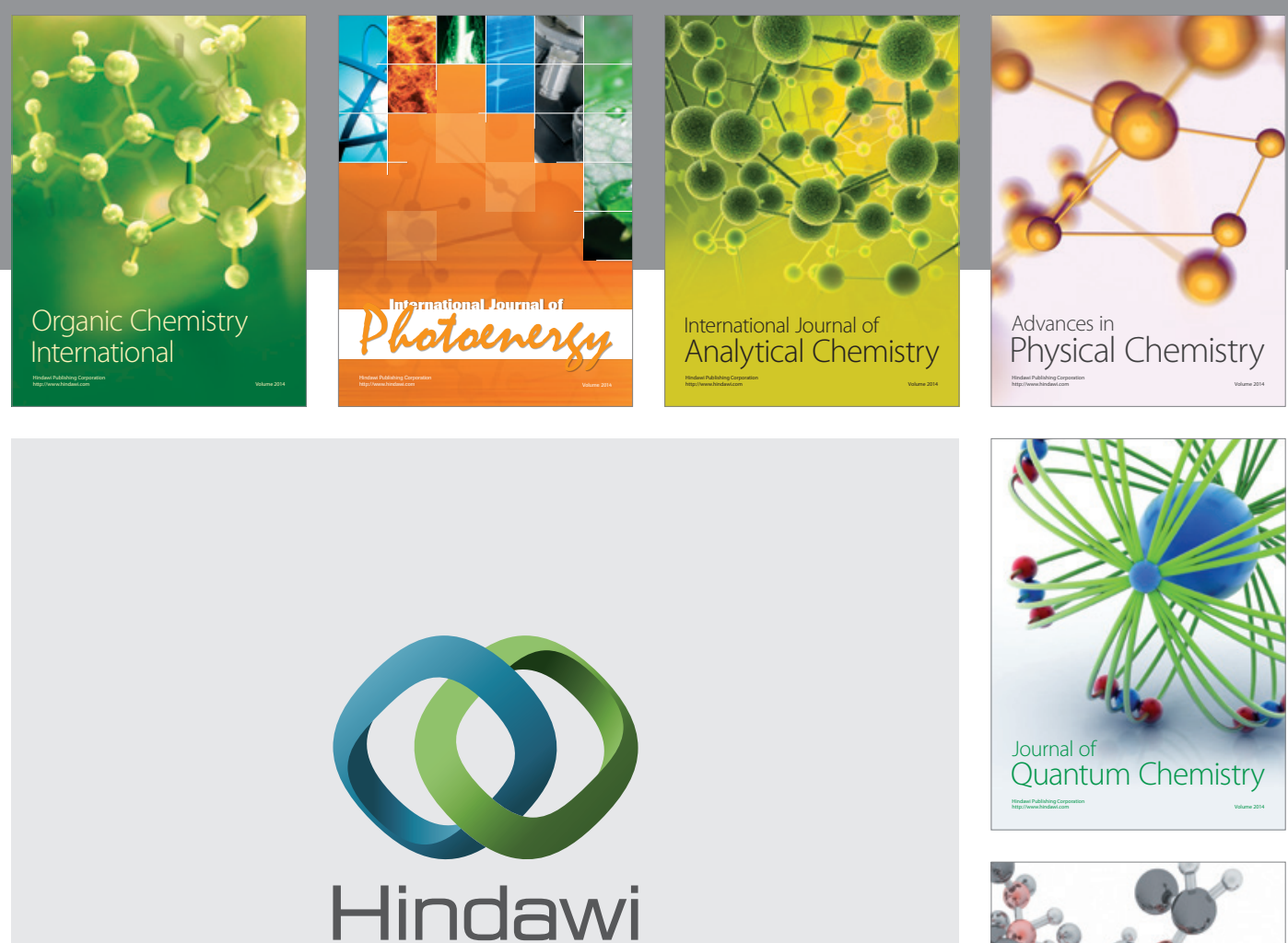

Submit your manuscripts at

http://www.hindawi.com

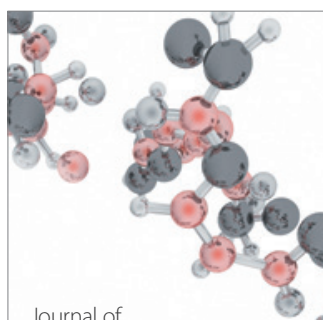

Analytical Methods

in Chemistry

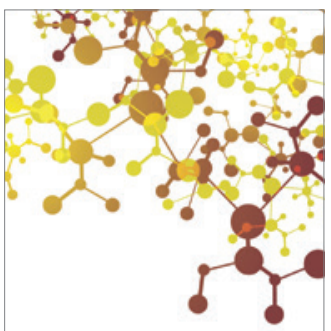

Journal of

Applied Chemistry

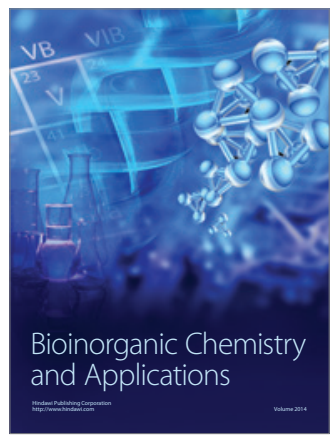

Inorganic Chemistry
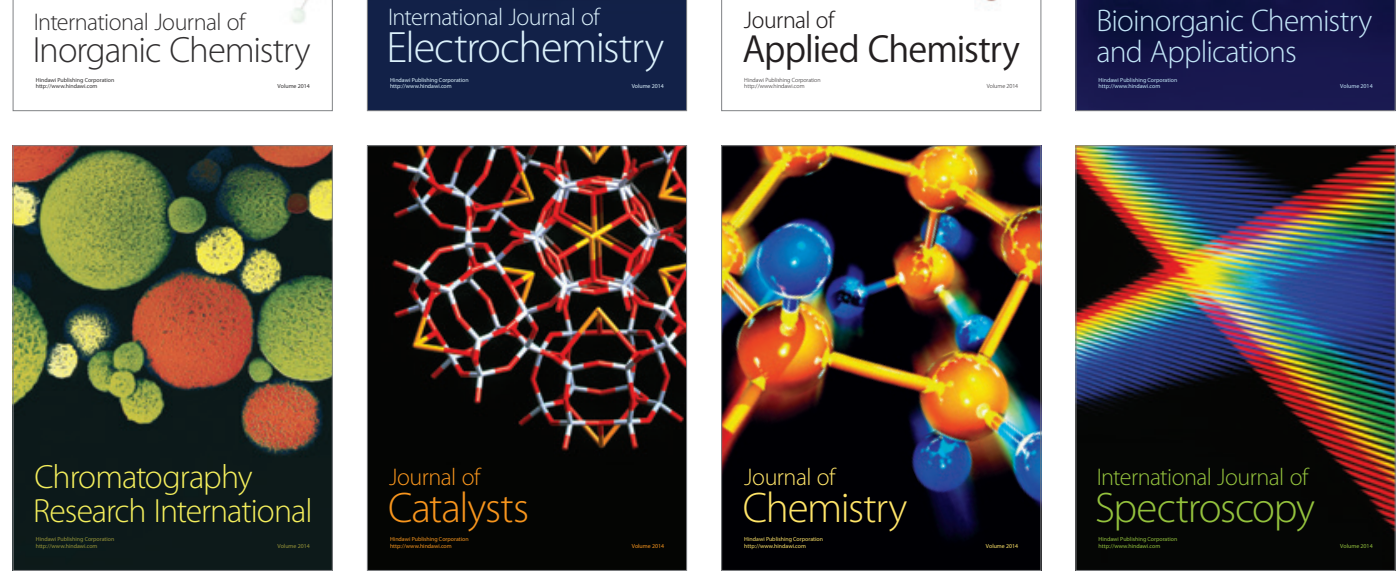